\title{
Perfil epidemiológico dos casos de gestantes com sífilis no estado da Bahia: 2014 a 2019
}

\author{
Epidemiological profile of cases of pregnant women with syphilis in the state of Bahia: \\ 2014 to 2019
}

Perfil epidemiológico de casos de mujeres embarazadas con sífilis en el estado de Bahia: 2014 a 2019

Greice Kely Oliveira de Souza ${ }^{1 *}$, Verena de Araújo Ribeiro Esquivel ${ }^{2}$, Cristiani de Souza Barros ${ }^{2}$, Myllena Ferreira Rabelo ${ }^{3}$, Érica Luzia Sales Murici de Jesus ${ }^{2}$, Osni Santos Paz ${ }^{2}$, Luciana Dourado Pimenta Almeida ${ }^{2}$, Itamara Queiroz dos Santos ${ }^{2}$, Alessandra Rabelo Gonçalves Fernandes², Tamyres Lopes Santana de Carvalho ${ }^{3}$.

\section{RESUMO}

Objetivo: Identificar o perfil das gestantes com sífilis, residentes no estado da Bahia, através da análise dos casos notificados no Sistema de Informação de Agravos e Notificações (SINAN). Métodos: Trata-se de um estudo ecológico de série temporal, descritivo e exploratório, realizado através de dados secundários fornecidos pelo SINAN. A população estudada foi constituída por gestantes que apresentaram sífilis no período de 2014 a 2019. Foram utilizadas as variáveis faixa etária, raça/cor, escolaridade, idade gestacional e classificação clínica. O estudo adotou a análise estatística descritiva, utilizando como ferramenta o software Microsoft Excel. Resultados: Entre os anos de 2014 e 2019, houveram 15.198 casos notificados de sífilis em gestantes, na Bahia, cujo ano com maior número de casos foi 2018, obtendo 3897 casos. A maior predominância dos casos foi entre gestantes na faixa etária de 20 a 29 anos, equivalendo $7.599(50 \%)$ casos, 9306 $(61,2 \%)$ declaravam-se parda, $3239(21,31 \%)$ possuíam a escolaridade de $5^{\underline{a}}$ a $8^{\text {a }}$ série incompleta, 5.027 (33\%) estavam no segundo trimestre gestacional, 4042 (27\%) foram classificados como sífilis primária. Conclusão: Os elevados números de casos de sífilis congênita entre gestantes, corrobora com desafios no diagnóstico precoce e o tratamento das gestantes, necessitando de medidas eficazes para prevenção.

Palavras-chave: Gravidez, Sífilis congênita, Doença sexualmente transmissível.

\begin{abstract}
Objective: To identify the profile of pregnant women with syphilis, living in the state of Bahia, through the analysis of the cases notified in the Diseases and Notifications Information System (SINAN) Methods: This is an ecological, time-series, descriptive and exploratory study, carried out using secondary dataa provided by SINAN. The studied population consisted of pregnant women who had syphilis from 2014 to 2019. The study adopted descriptive statistical analysis, using Microsoft Excel software as a tool. Results: Between 2014 and 2019 , there were 15,198 reported cases of syphilis in pregnant women in Bahia, whose year with the highest number of cases was 2018, obtaining 3897 cases. The greatest predominance of cases, $9306(61,2 \%)$ declared themselves brown, $3239(21.31 \%)$ had schooling from 5 to incomplete $8^{\text {th }}$ grade, $5,027(33 \%)$ were in the second trimester of pregnancy, $4042(27 \%)$ were classified as primary syphilis. Conclusion: The high number of cases of congenital syphilis among pregnant women, corroborates with challenges in the early diagnosis and treatment of pregnant women, in need of effective preventive measures.
\end{abstract}

Keywords: Pregnancy, Congenital syphilis, Sexually transmitted disease.

1 Universidade Estadual de Feira de Santana (UEFS), Feira de Santana - BA.

*E-mail: greicekely@hotmail.com.br

${ }^{2}$ Hospital Universitário Edgard Santos (HUPES), Salvador - BA.

${ }^{3}$ Faculdade Pitágoras de Feira de Santana, Feira de Santana - BA. 


\section{RESUMEN}

Objetivo: Identificar el perfil de las gestantes con sífilis residentes en el estado de Bahía, mediante el análisis de los casos notificados en el Sistema de Información de Enfermedades y Notificaciones (SINAN) Métodos: Se trata de un estudio ecológico, cronológico, descriptivo y exploratorio, realizado con datos secundarios proporcionados por SINAN. La población estudiada estuvo conformada por mujeres embrazadas que tenían sífilis de 2014 a 2019. Se utilizaron las variables edad, raza/color, educación, edad gestacional y clasificación clínica. El estudio adoptó un análisis estadístico descriptivo, utilizando como herramienta el software Microsoft Excel. Resultados: Entre 2014 y 2019 se reportaron 15.198 casos de sífilis en gestante en Bahía, cuyo año con mayor número de casos fue 2018, obteniendo 3897 casos. El mayor predominio de casos fue entre las gestantes de 20 a 29 años, equivalente a $7599(50 \%)$ casos, 9306(61,2\%) se declararon morenas, 3239 $(21,31 \%)$ tenían escolaridad de 5 a $8^{\circ}$ grado incompleto, $5027(33 \%)$ estaban en segundo trimestre del embarazo, 4042(27\%) se clasificaron como sífilis primaria. Conclusión: El alto número de casos de sífilis congénita entre gestantes, corrobora con desafíos en el diagnóstico y tratamiento precoz de estantes, necesita de medidas preventivas eficaces.

Palabras clave: Embarazo, Sífilis congénita, Enfermedad de trasmisión sexual.

\section{INTRODUÇÃO}

A Sífilis gestacional é uma enfermidade infecciosa causada pela bactéria gram-negativa Treponema Pallidum (SABACK MC, et al., 2019). Se não diagnosticada ou tratada durante a gestação pode ocasionar mortes fetais e neonatais precoces, sendo considerado um grave problema de saúde pública em nosso país (CESAR JA, et al., 2020). A transmissão da doença pode ser por via vertical, da mãe para o feto, pode ocorrer na passagem do canal do parto ou em qualquer momento da gestação ou estágio clinico da doença: durante o aleitamento materno, se houver lesões, na mama, através de gestantes que não foram tratadas ou das que realizaram tratamento de forma inadequada (OLIVEIRA JS e SANTOS JV, 2015).

A doença pode ser transmitida através de via sexual e vertical, sendo considerada sífilis adquirida e sífilis congênita, respectivamente. Existem outras formas de transmissão mais esporádica e que não são tão abordadas na literatura, devido um baixo interesse epidemiológico, como por exemplo: por via indireta (através de objetos contaminados com sangue) e por transfusão sanguínea (AVELLEIRA JCR, BOTTINO G, 2006). Apresenta múltiplos estágios: sífilis primária, secundária, latente e tardia ou terciária, essa divisão é realizada de acordo o tempo de evolução da doença. A sífilis primária manifesta-se por pequena ulceração, que é o cancro duro e surgimento de linfoadenomegalia inguinal entre duas e três semanas (MAGALHÃES DMS, et al., 2011).

A sífilis secundária ocorre em média entre seis semanas a seis meses após a cicatrização do cancro. Pode ocorrer em juntamente com a infecção primária e apresenta-se uma erupção macular eritematosa pouco visível (roséola), geralmente em tronco e raiz dos membros. A sintomatologia desaparece em algumas semanas, independentemente do tratamento. Na sífilis latente nãos e observa nenhum sinal nem sintoma. $O$ diagnóstico é exclusivamente através da reatividade dos testes treponêmicos e não treponêmicos (BRASIL, 2020).

A sífilis terciária pode se manifestar até 30 anos após a infecção inicial e caracteriza-se por lesões focalizadas que cobrem pele e mucosas, sistema cardiovascular e nervoso, marcado pela presença de granulomas destrutivos (conhecidos como gomas) e ausência quase total de treponemas (AVELLEIRA JCR, BOTTINO G, 2006).

Apesar das manifestações divergentes, o tratamento da patologia é feito através do uso de antibioticoterapia, independente da fase, tendo como droga de primeira escolha a Penicilina Benzatina. Mas para a eficácia, é necessário que o tratamento seja feito de forma adequada, havendo a necessidade de que o(s) parceiro(s) sexual(is) com sífilis sejam tratados, e que o tratamento seja realizado de acordo com o estágio, utilizando todas as doses prescritas (SOLINO MSS, et al., 2020).

A sífilis é considerada uma das infecções sexualmente transmissíveis que apresenta agravos importantes tanto às gestantes quanto aos seus conceptos, e por isso um grave problema de saúde pública. Mesmo que, 
tenha bem delimitada o agente causador da doença, os meios de contaminação e o tratamento, que é de baixo custo e alta efetividade (MAGALHÃES DMS, et al., 2011). A transmissão pode ocorrer em qualquer fase da gestação, porém nas fases primárias e secundárias, são períodos mais críticos devido as altas concentrações de espiroquetas circulantes na corrente sanguínea da gestação. Tem sido observado que 0 tratamento realizado antes da $20^{\mathrm{a}}$ semana de idade gestacional pode impedir a infecção do feto (ALBUQUERQUE GMA, et al., 2016).

O diagnóstico da sífilis é feito através de testes não treponêmicos, como: o Veneral Diseases Reserarch Laboratory (VDRL), Rapid Plasm Reagin (RPR); e de testes treponêmicos: Enzime-linked Immunosorbent Assay (Elisa), Treponema pallidum Hemagglutination (TPHA) e Fluorescent Treponemal Antibody Absorption (FTA-abs). Dentre os testes, o não treponêmico é o mais utilizado, pois apresenta uma elevada sensibilidade, simples, rápido e baixo custo (MOTA IA, et al., 2018).

De acordo com as estatísticas, o continente Americano apresenta a segunda maior prevalência de sífilis durante o período gestacional e a terceira maior em número de casos. Nesse ínterim, A Organização PanAmericana de Saúde tem como meta a eliminação da sífilis congênita nas Américas, definida como a ocorrência de menos de 0,5 casos para cada 1000 nascidos vivos. Essa meta é adotada pelo Ministério da Saúde no Brasil (DOMINGUES RMSM, et al., 2014). O Brasil é um dos países prioritários para o controle da sífilis congênita devido ao seu tamanho populacional e prevalência da sífilis gestacional (BRASIL, 2014).

Em relação à realidade brasileira, configura-se uma epidemia, com incidência de 6,5/1000 nascidos vivos. No período de 2010 a 2019, foram notificados no Sistema de Informações de Agravos de Notificação (SINAN) 332.951 casos de sífilis em gestantes no Brasil (BRASIL, 2016; BRASIL, 2020).

Contudo, estima-se que o número de casos de sífilis gestacional real seja maior que o número de casos notificados. Este fato pode estar relacionado com dificuldades no diagnóstico e/ou na notificação deste agravo. Estudos nacionais apontam falhas na assistência pré-natal relativas ao controle de sífilis na gestação, com oportunidades perdidas de controle e tratamento. O diagnóstico oportuno da sífilis na gravidez é o principal desafio para o controle da Sífilis Congênita (ROSA RFN, et al., 2020).

Assim sendo, diante do exposto, o presente estudo tem como objetivo identificar o perfil das gestantes com sífilis, residentes no estado da Bahia, através da análise dos casos notificados no Sistema de Informação de Agravos e Notificações (SINAN). Visando colaborar com a elaboração de ações e programas eficazes de assistência e promoção à saúde, que corroborem com o diagnóstico e tratamento precoce da sífilis gestacional, consequentemente seus efeitos sobre o concepto.

\section{MÉTODOS}

Trata-se de um estudo ecológico de série temporal, descritivo e exploratório, realizado através de dados secundários fornecidos pelo Sistema de Informação de Agravos de Notificação (SINAN). O estudo ecológico visa o conhecimento a cerca de uma determinada população, conjunto de indivíduos. Não é possível reconhecer a particularidade de casa indivíduo (FILHO JGB, et al., 2012).

Por tratar-se de uma pesquisa que utiliza informações públicas, essa pesquisa se isenta da necessidade de aprovação prévia do Comitê de Ética em Pesquisa, respeitando as premissas contidas nas resoluções 466/12 e 510/16 do Conselho Nacional de Saúde.

O SINAN é um sistema de informação que coleta e fornece dados gerados pelo Sistema de Vigilância Epidemiológica brasileiro sobre doenças e agravos da lista nacional de notificação compulsória. $O$ Departamento de Informática do Sistema Único de Saúde (DATASUS) permite análises da situação sanitária contribuindo para tomadas de decisão baseadas em evidências e subsídio ou manutenção de programas de saúde.

A população estudada foi constituída por gestantes que apresentaram sífilis no período de 2014 a 2019. O Sistema de Informação de Agravos de Notificação (SINAN), foram as fontes de dados do estudo. As variáveis analisadas foram: idade, raça/cor, escolaridade, idade gestacional e classificação clínica. O estudo adotou a análise estatística descritiva, utilizando como ferramenta o software Microsoft Excel®. 
A Bahia é o maior estado da região Nordeste, sua área territorial é de $564.760,427 \mathrm{~km}^{2}$, possui aproximadamente 14.930.634 habitantes (IBGE, 2020). Além disso, é rica em diversidade cultural e ambiental, possuindo diversos pontos turísticos, incluindo a sua capital Salvador, que foi a primeira capital do Brasil.

\section{RESULTADOS}

Entre os anos de 2014 e 2019, houveram 15.198 casos notificados de sífilis em gestantes, na Bahia. Observou-se um crescimento no número de casos notificados e sífilis em gestante, elevando-se de 1747 para 3897, entre os anos e 2014 a 2018. Onde sobressaiu-se o ano de 2018, obtendo 3897 casos, havendo um decréscimo no ano de 2019, sendo identificado 2274 notificações (Figura 1).

Figura 1 - № de casos de gestantes com sífilis na Bahia, 2014 - 2019.

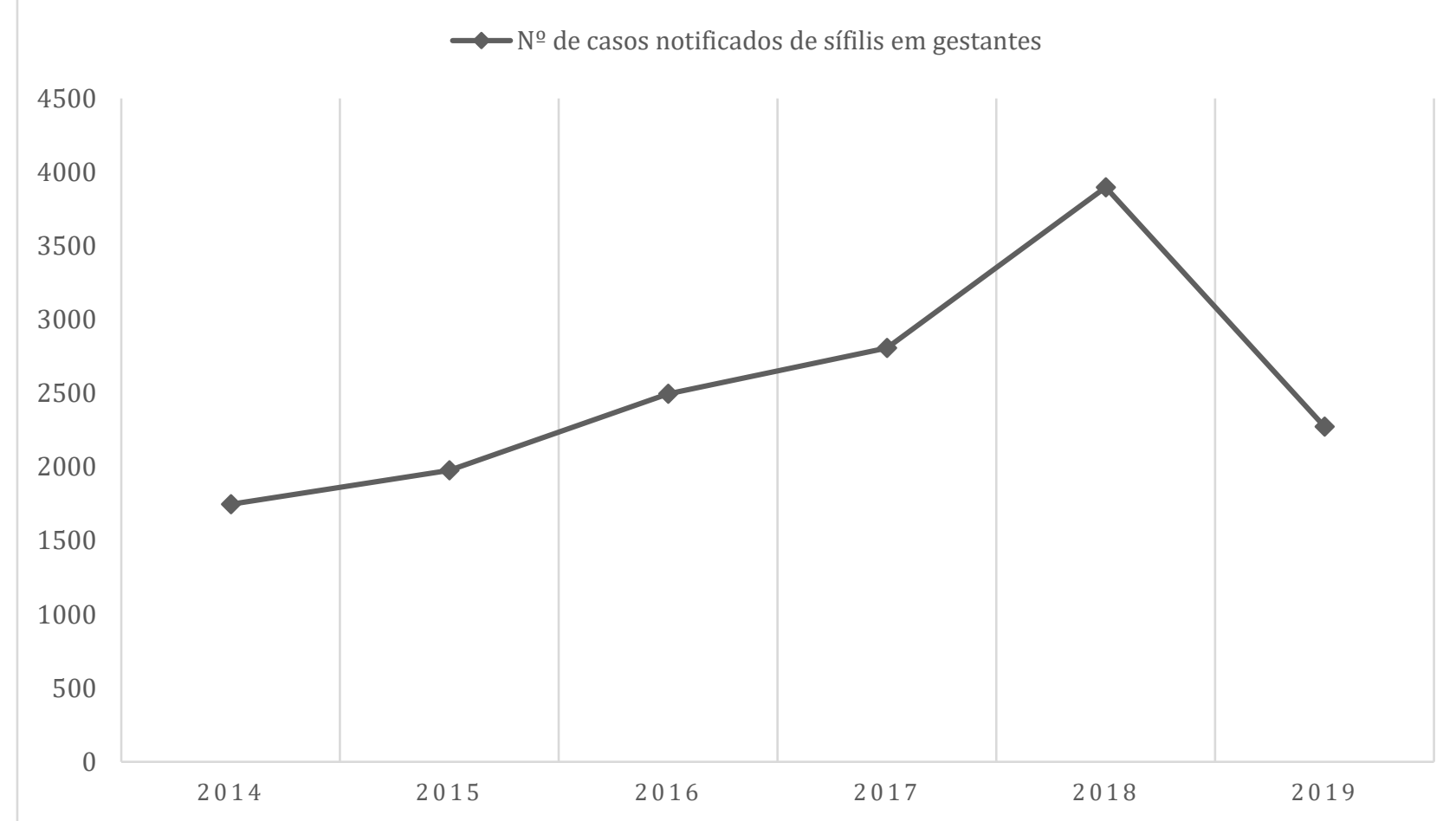

Fonte: Souza GKO, et al., 2020; dados extraídos do Sistema de Informação de Agravo e Notificação - SINAN/ DATASUS.

De acordo com as características maternas, observa-se maior predominância na faixa etária de 20 a 29 anos, equivalendo 7.599 casos, $50 \%$ dos casos do período analisado, subsequente daquelas de 15 a 19 anos, obtendo $3660(24 \%)$ casos, 30 a 39 anos $3338(22 \%)$ casos, de idade maior ou igual a 40 anos foram 382 (3\%) casos, de 10 a 14 anos $215(1 \%)$ casos. No que se refere a raça/cor, $9306(61,2 \%)$, declaravam-se parda, $3175(20,9 \%)$ negras, $1056(6,9 \%)$ brancas, $151(1 \%)$ amarelas, $73(0,5 \%)$ indígenas, 1437 foram ignoradas, caracterizando $9,5 \%$ dos casos.

Houve maior número de casos entre aquelas que possuíam a escolaridade de $5^{\mathrm{a}}$ a $8^{\mathrm{a}}$ série incompleta, configurando $3239(21,31 \%)$ dos casos, $2087(13,74 \%)$ possuíam o médio completo, $1702(11,20 \%)$ médio incompleto, $1052(6,92 \%) 1^{\text {a }}$ a $4^{\text {a }}$ série incompleta, $1026(6,75 \%)$ fundamental completo, $627(4,13 \%)$ casos $4^{\text {a }}$ série completa, $150(0,99 \%)$ eram analfabetas, $133(0,88 \%)$ superior incompleto, $91(0,60 \%)$ superior completo, $3(0,02 \%)$ não se aplica e 5087 (33,47\%) tiveram a escolaridade ignorada.

No que diz respeito ao período gestacional em que houve identificação da patologia, 3800 (25\%) casos foram notificados no $1^{\circ}$ trimestre, $5.027(33 \%)$ no segundo trimestre, $4891(32 \%)$ no terceiro trimestre, 1.480 (10\%) ignoraram a idade gestacional. Quanto as classificações clínicas, 4946 (33\%) casos tiveram a classificação clínica ignorada, 4042 (27\%) foram classificados como sífilis primária, 884 (6\%) obtiveram sífilis secundária, 2186 (14\%) sífilis terciária, 3.140 (21\%) corresponderam a sífilis latente (Tabela 1). 
Tabela 1- Caracterização das gestantes com sífilis. Bahia 2014-2019.

\begin{tabular}{|c|c|c|}
\hline Variável & $\mathbf{N}$ & $\%$ \\
\hline \multicolumn{3}{|l|}{ Idade } \\
\hline $10-14$ & 215 & 1 \\
\hline $15-19$ & 3.660 & 24 \\
\hline $20-29$ & 7.599 & 50 \\
\hline $30-39$ & 3.338 & 22 \\
\hline $40+$ & 382 & 3 \\
\hline \multicolumn{3}{|l|}{ Raça/cor } \\
\hline Branca & 1.056 & 6,9 \\
\hline Preta & 3.175 & 20,9 \\
\hline Amarela & 151 & 1,0 \\
\hline Parda & 9.306 & 61,2 \\
\hline Indígena & 73 & 0,5 \\
\hline Ignorada & 1.437 & 9,5 \\
\hline \multicolumn{3}{|l|}{ Escolaridade } \\
\hline Analfabeto & 150 & 0,99 \\
\hline $1^{\text {a }}$ a $4^{\mathrm{a}}$ Série Incompleta & 1.052 & 6,92 \\
\hline 4aㅗ Série Completa & 627 & 4,13 \\
\hline $5^{\text {a }}$ a $8^{\mathrm{a}}$ Série Incompleta & 3.239 & 21,31 \\
\hline Fundamental Completo & 1.026 & 6,75 \\
\hline Médio Incompleto & 1.702 & 11,20 \\
\hline Médio Completo & 2.088 & 13,74 \\
\hline Superior Incompleto & 133 & 0,88 \\
\hline Superior completo & 91 & 0,60 \\
\hline Não se aplica & 3 & 0,02 \\
\hline Ignorado & 5.087 & 33,47 \\
\hline \multicolumn{3}{|l|}{ Idade Gestacional } \\
\hline 1ㅇ Trimestre & 3.800 & 25 \\
\hline 2o Trimestre & 5.027 & 33 \\
\hline 3o Trimestre & 4.891 & 32 \\
\hline Idade Gestacional Ignorada & 1.480 & 10 \\
\hline \multicolumn{3}{|l|}{ Cassificação Clínica } \\
\hline Sífilis Primária & 4.042 & 27 \\
\hline Sífilis Secundária & 884 & 6 \\
\hline Sífilis Terciária & 2.186 & 14 \\
\hline Sífilis Latente & 3.140 & 21 \\
\hline Ignorado & 4.946 & 33 \\
\hline
\end{tabular}

\section{DISCUSSÃO}

Entre os anos de 2014 a 2019, observou-se que a maioria dos casos notificados de gestantes com sífilis, na Bahia, ocorreram entre jovens adultas, declaradas parda e com baixo nível de escolaridade. Notou-se também que os diagnósticos, em sua maior parte, foram realizados no segundo trimestre gestacional, e classificadas como sífilis primária. 
Durante o período, tiveram 15.198 novos diagnósticos de sífilis em gestantes no estado da Bahia, onde 7.599 (50\%) dos casos acometeu a faixa etária de 20 a 29 anos, devido aos fatores de vulnerabilidades que são mais suscetíveis. Em 2016 e 2018 ocorreram os maiores números de casos, apresentando uma redução considerável em 2019. Em relação a variável raça/cor, prevaleceu a cor parda 9.306 (61,2\%), como também indivíduos com fundamental incompleto 3.239 (21,31\%). A maioria dos casos foram notificados no segundo trimestre de gestação compreendendo 5.027 (33\%). Tais desigualdades já haviam sido pontuadas para sífilis (BERTUSSO TCB, et al., 2018), sendo corroboradas pelos presentes resultados, que adentram em uma reflexão ao revelar que mulheres pretas e pardas, de baixa escolaridade, apresentavam maiores prevalências para a infecção.

Este estudo demonstra que a sífilis durante a gestação apresenta alta incidência e que é um grande problema de saúde pública, visto que, há probabilidade de desenvolver sífilis no neonato, mesmo sendo uma doença tratável e curável. No Brasil, a taxa de detecção da sífilis em gestantes vem aumentando: em 2010 havia 3,5 casos de sífilis gestacional (SG) e 2,4 casos de Sífilis Congênita (SC) para cada 1.000 nascidos vivos; em 2016 as taxas de detecção de SG e incidência de SC foram, respectivamente, de 12,4 e 6,8 casos para cada 1.000 nascidos vivos (BRASIL, 2017).

O perfil encontrado das gestantes com sífilis no estudo está em consonância com os resultados encontrados na literatura, tanto em nível nacional como internacional e reafirma a forma como a doença se comporta em adultos jovens que estão no ápice da fase reprodutiva, com o nível educacional baixo (QIN JB, et al., 2014; DOMINGUES RMSM, et al., 2014; CAVALCANTE PAM, et al., 2017).

Segundo Cavalcante PAM, et al. (2017), o diagnóstico indolente deste agravo, pode estar relacionado ao momento que é iniciado o pré-natal, e baixa qualidade da assistência fornecida para as gestantes, uma vez que as consultas regulares e precoces contribuem significativamente para a detecção da sífilis, consequentemente com a efetividade do tratamento.

Entre os principais fatores que podem ter contribuído para tal cenário, podemos destacar que, a partir de 2014, tanto no Brasil como no exterior, houve desabastecimento da penicilina benzatina (PB) devido à deficiência na matéria-prima específica para produzi-la. Entre as causas da falta de penicilina no mundo é a dependência de um pequeno número de fornecedores globais do insumo farmacêutico ativo (IFA). Nos últimos dez anos ao menos cinco empresas abandonaram o mercado global de penicilina em busca de medicamentos que gerassem maior lucro (ARAÚJO RS, et al., 2020).

É extremamente reconhecida a eficácia de diagnóstico, tratamento e prevenção da transmissão (NEWMAN L, et al., 2013), a taxa de gestantes que são infectadas com sífilis que não realizam terapia e intervenção sobre os fatores de risco ainda é alta, o que gera complicações graves, como: aborto, prematuridade, óbito neonatal e malformações congênitas.

No que concerne a classificação clínica da sífilis, observa-se que a sífilis primária corresponde a $24,2 \%$, sífilis secundária $5,6 \%$, sífilis terciaria $14,2 \%$, sífilis latente $21 \%$ e ignorado $32,8 \%$. De acordo o Ministério da Saúde, existe uma dificuldade na detecção da sífilis em gestantes, uma vez que o cancro duro é assintomático e se localiza em regiões que dificulta a sua visualização, como: cervix, períneo ou parede vaginal. Destoando do que se espera no rastreamento, a fase primária apresentou uma maior incidência, esse dado sugere que pode ter ocorrido equivocadamente a classificação da doença. Ressalta-se que erros de interpretação e classificação da fase clínica ocasionam tratamentos inadequados e, portanto, devem ser classificados como ignorada, quando não for possível estabelecer a evolução da doença, sendo preconizado o tratamento com três doses de penicilina benzatina (BRASIL, 2015).

O ano que apresentou o maior número de casos foi 2018, apresentando um decréscimo considerável no ano de 2019. Divergindo do que reflete estudos em nível nacional, os quais apresentam aumento significativo dos casos de sífilis na gestação a cada ano. $O$ dado isolado de redução considerável de casos notificados no ano de 2019 pode não refletir redução direta de incidência de sífilis gestacional, podendo ter havido subnotificações dos casos, com necessidade, portanto de avaliação de outras variáveis para a conclusão de queda de números absolutos de sífilis em gestantes (LAFETÁ KRG, et al., 2016). 
A ocorrência das subnotificações impede o controle epidemiológico do agravo, indo no sentido contrário as políticas públicas atuais, especialmente da Rede Cegonha, pois além do aperfeiçoamento na área da saúde obstétrica-neonatal, contribuem para o aprimoramento do sistema de vigilância epidemiológica, através do acréscimo na distribuição de testes rápidos para sífilis, ferramenta que permite o rastreio do agravo (NEWMAN L, et al., 2013).

Segundo Domingues RMSM, et al. (2014), esse aumento gradativo sugere dificuldades no diagnóstico e/ou na notificação deste agravo. Estudos nacionais apontam falhas na assistência pré-natal relativas ao controle de sífilis na gestação, como oportunidades perdidas para controle e tratamento. Mulheres de menor escolaridade, pretas ou pardas, e aquelas atendidas em serviços públicos tiveram as coberturas mais baixas de testagem e foram as que apresentaram maior prevalência de sífilis na gestação. Isso sugere oportunidades perdidas de diagnóstico e intervenção justamente nas mulheres com maior risco de transmissão vertical da sífilis (BOTTURA BR, et al., 2019).

A presença da sífilis congênita é um marcador que ocorreram déficits desde o pré-natal, de modo que a deve-se investir em ações preventivas e protetoras (MAGALHÃES DMS, et al., 2011). Contudo, em concordância com estudos nacionais, a maior prevalência de sífilis na gestação encontrada em mulheres com menor escolaridade, de cor da pele preta ou parda, sem assistência pré-natal e atendidas em serviços públicos sugere que são esses os grupos populacionais mais expostos a essa infecção, devendo ser objeto de maior atenção dos programas de controle da sífilis na gestação. Cabe ressaltar que a baixa escolaridade é um fator de risco considerável para exposição às infecções sexualmente transmitidas, devido a restrição do entendimento das medidas de prevenção (SOARES LG, et al., 2017; CAVALCANTE PAM, et al., 2017).

Vale salientar, que a inclusão do parceiro durante o pré-natal institui uma estratégia importante para o controle da infecção. Dessa forma, há necessidade de melhorar o acesso aos serviços de saúde, também, para os homens, assim como estimular a valorização do cuidado da saúde destes. De acordo com Qin JB, et al. (2014), há denotação de que parceiros infectados com sífilis aumenta em cinco vezes a chance de sífilis congênita e as justificativas para não realizar o tratamento são: reduzida adesão aos serviços de saúde; questões empregatícias e falta de conhecimento sobre a transcendência do tratamento para saúde da criança e da parceira; falta de local de referência para tratamento; e a não indicação do tratamento pelo serviço (FRANÇA ISX, et al., 2015).

A notificação tardia desses casos pode refletir algumas deficiências do sistema de saúde, desde o acesso da população a um pré-natal adequado, até a dificuldade de estabelecer diagnóstico e exames, implicando diretamente na eficácia do tratamento e prevenção consequente da sífilis congênita. Assim, tais resultados corroboram para a importância do diagnóstico e do tratamento oportuno da gestante e de seu (s) parceiro (s). Para tanto, são imprescindíveis medidas de conscientização tanto da mãe quanto de seu (s) companheiro (s) sobre a doença, formas de contágio e suas repercussões, tanto para o feto quanto para a genitora. Somado a isso, é de extrema importância a atualização dos profissionais da saúde sobre o assunto, para que haja um manejo adequado dos casos e uma melhor notificação, com o objetivo de acompanhar de forma correta o comportamento de tal doença e, assim, fazer um planejamento efetivo para seu tratamento e prevenção e, sobretudo, para a melhoria da assistência.

Diante do exposto, faz-se necessário investimento na atenção básica, tanto para capacitação profissional, quanto no acesso dessas gestantes à assistência pré-natal adequada. Além de proporcionar educação em saúde, seja em ambiente escolar, como em serviços de saúde, garantindo acesso a informação, proporcionando conhecimento sobre saúde sexual. Aliado a atenção primária em saúde, os agentes comunitários de saúde, podem ser de extrema importância quanto ao acesso direto a essa população, podendo ser agente identificador de grupo de risco, população mais vulnerável e supervisão de adesão ao tratamento, tanto das gestantes quanto dos seus parceiros, estes últimos geralmente mais resistentes ao tratamento e responsáveis por reinfecção das gestantes.

Este estudo demonstrou algumas limitações, inerentes às pesquisas com dados secundários, devido a possibilidade de subnotificações. Os resultados apresentados levam à constatação da fragilidade dos serviços de saúde quanto ao controle da sífilis gestacional e consequentemente congênita. 


\section{CONCLUSÃO}

A sífilis congênita, apesar de haver formas de prevenção, é uma patologia aumenta gradativamente, sem afinidade específica de faixa etária, raça ou classe social. Entretanto, observou-se que as mulheres mais afetadas, são jovens adultas, declaradas pardas e com baixo nível de escolaridade. O principal desafio para o controle da sífilis congênita é o diagnóstico precoce e o tratamento das gestantes, para tanto é necessário, universalizar a oferta de cuidados. Percebe-se a necessidade de implementar medidas mais eficazes no contexto profissional e melhoria nos serviços de saúde para a realização do manejo adequado. Um início tardio de assistência pré-natal constitui uma barreira para o controle da sífilis gestacional, estando diretamente relacionado a um menor número de consultas e a menor realização de exames de rotina. Mesmo sendo de extrema importância e impacto na saúde pública, com grande quantidade de estudos publicados acerca da qualidade do cuidado pré-natal e das características marcadoras das vulnerabilidades, a meta para controle da doença estabelecida há alguns anos ainda não foi alcançada. Contudo, o estudo apresentou como limitações artigos atuais que versassem sobre o tema, demonstrando a importância de publicações científicas na área e a análise ecológica, por utilizar medidas agrupadas, denota importante fonte de incerteza na inferência ecológica, devido a impossibilidade de associação entre exposição e doença no nível individual.

\section{REFERÊNCIAS}

1. ALBUQUERQUE GMA, et al. Complicações da sífilis congênita: uma revisão de literatura. Pediatria Moderna, 2014; 50(6): 254-258

2. ARAÚJO RS, et al. A quem afetou o desabastecimento de penicilina para sífilis no Rio de Janeiro, 2013-2017? Rev Saúde Pública, 2020; 54: 109.

3. AVELLEIRA JCR, BOTTINO G. Sífilis: diagnóstico, tratamento e controle. An Bras Dermatol. 2006; 81(2):111-26.

4. BERTUSSO TCB, et al. Características de gestantes com sífilis em um hospital universitário do Paraná. R. Saúde Públ., 2018; 1(2): 129-140.

5. BOTTURA BR, et al. Perfil epidemiológico da sífilis gestacional e congênita no Brasil - período de 2007 a 2016 . Arq Med Hosp Fac Cienc Med Santa Casa São Paulo, 2019; 64(2): 69-75.

6. BRASIL. 2014. Boletim Epidemiológico DST/AIDS e Hepatites Virais. Governo do Estado do Rio de Janeiro. Disponível em: http://www.riocomsaude.rj.gov.br/Publico/MostrarArquivo.aspx?C=sF6igzRT/p4=. Acesso em: 20/01/2021

7. BRASIL. Boletim Epidemiológico da Sífilis. Brasília. Ministério da Saúde. Secretaria de Vigilância em Saúde. Departamento de DST, Aids e Hepatites Virais, 2016; 35: 47.

8. BRASIL. Protocolo clínico e diretrizes terapêuticas para atenção integral às pessoas com infecções sexualmente transmissíveis. Brasília. Ministério da Saúde. Secretaria de Vigilância em Saúde. Departamento de DST, Aids e Hepatites Virais, 2015.

9. BRASIL. Protocolo Clínico e Diretrizes Terapêuticas para Atenção Integral às Pessoas com Infecções Sexualmente Transmissíveis (IST). Brasília: Ministério da Saúde. Secretaria de Vigilância em Saúde. Departamento de Doenças de Condições Crônicas e Infecções Sexualmente Transmissíveis. 2020.

10. BRASIL. Sífilis. Bol Epidemiol. Ministério da Saúde. Secretaria de Vigilância em Saúde, 2017; 48(36): 4-41. Disponível em: http://www.aids.gov.br/pt-br/pub/2017/boletim-epidemiologico-de-sifilis-2017. Acesso em: 05/11/2020

11. CAVALCANTE PAM, et al. Sífilis gestacional e congênita em Palmas, Tocantins, 2007-2014. Epidemiol Serv Saúde, 2017; 26 (2): 255-64.

12. DOMINGUES RMSM, et al. Prevalência de sífilis na gestação e testagem pré-natal: Estudo Nascer no Brasil. Rev Saúde Pública, 2014; 48 (5): 766-74.

13. FRANÇA ISX, et al. Fatores associados à notificação da sífilis congênita: um indicador de qualidade da assistência pré-natal. Rev Rene, 2015; 16 (3): 374-81.

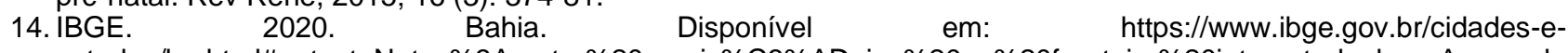
estados/ba.html\#: :text=Notas\%3A,entre\%20munic\%C3\%ADpios\%20na\%20fronteira\%20interestadual. Acessado em: 05/02/2021

15. LAFETÁ KRG, et al. Sífilis materna e congênita, subnotificação e difícil controle. Rev Bras Epidemiol, 2016; 19(1): 6374.

16. MAGALHÃES DMS, et al. Sífilis materna e congênita: ainda um desafio. Cad Saúde Pública, 2013; 29(6): 1109-1120.

17. MOTA IA, et al. Sífilis congênita: por que sua prevalência continua tão alta? Rev Med Minas Gerais, 2018; 28(6).

18. NEWMAN L, et al. Global Estimates of Syphilis in Pregnancy and Associated Adverse Outcomes: Analysis of Multinational Antenatal Surveillance Data. PLoS Med, 2013; 10(2).

19. QIN JB, et al. Maternal and paternal factors associated with congenital syphilis in Shenzhen, China: a prospective cohort study. Eur J Clin Microbiol Infect Dis, 2014; 33 (2): 221-32.

20. ROSA RFN, et al. O manejo da sífilis gestacional no pré-natal. Rev enferm UFPE on line, 2020; 14: e243643.

21. SOARES LG, et al. Sífilis gestacional e congênita: características maternas, neonatais e desfecho dos casos. Rev. Bras. Saúde Mater. Infant., 2017; 17(4): 781-789.

22. SOLINO MSS, et al. Desafios do enfermeiro na assistência de enfermagem aos usuários com diagnóstico de sífilis: revisão integrativa. Braz. J. Hea. Rev., 2020; 3(5): 13917-13930. 